\title{
Earthquake economic loss assessment of gas system: Ningbo as an example
}

\author{
Ganhua Fang ${ }^{1}$, Shaoqing $\mathrm{Hu}^{1,{ }^{*}}$, Xin $\mathrm{Chen}^{1}$, Weiwei $\mathrm{Xu}^{1}$ \\ ${ }^{1}$ School of Architecture and Civil Engineering, Ningbo University of Technology, Ningbo, 315016, \\ China \\ *Flywing0518@163.com
}

Keywords: Gas pipeline; earthquake damage; repair cost; economic loss assessment

\begin{abstract}
City Gas system is an important urban infrastructure. Historical earthquakes show gas system is vulnerable in the earthquake. Earthquake damage of the gas system is mainly pipeline damage. Damage usually concentrated in low-pressure pipe and tube into the household, especially the joint pulling out or pulling off and pipeline rupture. How to assess the economic loss quickly of gas system after earthquake is an urgent problem. By collating earthquake damage data of gas system, the characteristics of earthquake damage are analyzed. The investigations of the pipeline repair cost and the broken road repair cost are carried out in Ningbo City. Then the repair costs for different diameters of different gas pipes are reasonably given. The results provide a reasonable data of earthquake economic losses evaluation for the gas pipeline system.
\end{abstract}

\section{Introduction}

With the development in our country, more and more urban use gas. Gas pipeline and affiliated facilities became part of modern life, which is called the city lifeline engineering. As a result, some areas and towns need to seriously consider that the earthquake damage and loss of gas system are more serious for town more modernization, population more concentrated, gas system more perfect, earthquake damage and loss is more serious. The gas supply facilities suffered different degree damage in Wenchuan earthquake.

It is efficiency for the earthquake emergency rescue work to timely and accurate acquaint with the seismic loss and its distribution after strong earthquake. The earthquake emergency rescue work practice in Wenchuan 8.0 earthquake showed that the seismic damage assessment technology of gas pipeline can not fully meet the actual demand. In this paper, through the investigation of the earthquake damage and the repair cost in earthquake loss assessment model, then the earthquake disaster loss evaluation method can be improved. It can be provided some intentional reference and advice for the earthquake disaster loss assessment methodology.

\section{The seismic damage factors of gas pipeline}

All previous earthquake damage investigation results show that gas pipeline network could be significantly damaged in the seismic intensity region for VII and VII degrees above. The reasons for earthquake damage of gas pipeline are mainly the inertia force due to the earthquake ground motion and other loads for the pipe on the ground. And for underground pipeline, the first reason is the ground damage, such as permanent seismic surface (rose and subsidence, ground fault rupture, etc.) caused by structural motion, and sand liquefaction landslides, uneven settlement and so on caused by the ground motion. The second reason is the relative displacement caused by seismic waves spreading. A large number of investigation showed that the most serious damage to buried pipeline is caused by surface rupture due to the fault, followed by sand liquefaction and uneven settlement. It is the most common for damage of buried pipeline is caused by the strong ground motion.

The main damage forms of buried pipeline are interface damage, the tube body destruction, connection destruction and the tub collapsed due to landslide and the influence of strong earthquakes. 


\section{The Seismic vulnerability model of gas pipeline}

According to the historical seismic damage statistics results of gas pipeline and considering the practical situation that the gas pipeline are severely damaged and need to be rebuilt when subjected to the seismic intensity of $\mathrm{X}$ degree and above, the average damage rate of buried gas pipeline under different seismic intensity are given, as shown in table 1-1.

Tab.1-1 The average damage rate of buried gas pipeline (one point $/ \mathrm{km}$ )

\begin{tabular}{cccccc}
\hline Intensity & 6 degree & 7 degree & 8 degree & 9 degree & Above 10 degree \\
\hline Steel pipe & 0.03 & 0.38 & 1.07 & 5.00 & - \\
PE pipe & 0.00 & 0.03 & 0.32 & 1.05 & - \\
“_” & showed that gas pipeline suffer serious damage and need to rebuild.
\end{tabular}

\section{Earthquake disaster loss assessment method of gas pipeline}

\section{The repair cost of one damage point of gas pipeline.}

The loss ratio of the general structure refers to the ratio between the repair cost and replacement cost, expressed by the percentage of $0-100 \%$. For gas pipeline system, due to the wide distribution, the pipe diameter and pipe material are different in different. So the earthquake damage loss calculation of pipeline should be based on the repair cost of each damage point not the whole network as a unit. The direct earthquake economic losses of gas pipeline are mainly focused on the post-disaster repair costs, including the broken road repair cost and the pipe itself repair cost. According to the post earthquake reconstruction project statistics of different cities, the line length of the broken road is approximately 4 meters. Thus, the repair cost formula of one point of gas pipeline is given as the following.

$$
G_{i}=\left(g_{1}+g_{2}\right) \times \beta
$$

$g_{1}$ _ the broken road repair cost of each point of gas pipeline; (Ten thousand yuan/ one point)

$g_{2}$ _ the pipe itself repair cost of each point of gas pipeline ; (Ten thousand yuan/ one point)

$\beta$ - Construction difficulty coefficient, the value is 1.1 in adverse site, otherwise is 1.0.

Generally, the pipe of urban gas pipeline can be used L series of steel pipe, Q235B pipe, 20\# pipe, PE pipe, cast iron pipe, etc. In this paper mainly according to the repair cost data of DN50 DN1000 diameter tubes in Ningbo City, the broken road repair cost under different categories of roads and the pipe itself repair cost are given, as shown in Table 1-2 to table 1-7 shows respectively.

Tab.1-2 The broken road repair cost of different diameter pipe under different categories

\begin{tabular}{ccccc} 
& \multicolumn{4}{c}{ [Ten thousand yuan/ on } \\
\hline \multirow{2}{*}{ pipe diameter } & Dirt road & different categories road & \\
& 0.14 & 0.46 & 0.67 & 0.83 \\
Under DN50 & 0.15 & 0.47 & 0.69 & 0.85 \\
DN100 & 0.15 & 0.49 & 0.71 & 0.88 \\
DN150 & 0.16 & 0.50 & 0.74 & 0.91 \\
DN200 & 0.17 & 0.54 & 0.78 & 0.96 \\
DN300 & 0.18 & 0.57 & 0.82 & 1.02 \\
DN400 & 0.26 & 0.81 & 1.18 & 1.45 \\
DN500 & 0.27 & 0.85 & 1.23 & 1.52 \\
DN600 & 0.28 & 0.88 & 1.29 & 1.59 \\
DN700 & 0.29 & 0.92 & 1.34 & 1.66 \\
DN800 & 0.32 & 1.00 & 1.45 & 1.80 \\
DN1000 & & & &
\end{tabular}


Tab. 1-3 The pipe itself repair cost of different pipe diameter ( $\mathrm{L}$ series of steel pipe)

[Ten thousand yuan/ one point]

\begin{tabular}{ccccc}
\hline \multirow{2}{*}{ pipe diameter } & \multicolumn{4}{c}{ Pressure level } \\
\cline { 2 - 5 } & $\begin{array}{c}\text { Sub high pressure B } \\
(1.0 \mathrm{Mpa})\end{array}$ & $\begin{array}{c}\text { sub high pressure A } \\
(1.6 \mathrm{Mpa})\end{array}$ & $\begin{array}{c}\text { high pressure B } \\
(2.5 \mathrm{Mpa})\end{array}$ & $\begin{array}{c}\text { high pressureA } \\
(4.0 \mathrm{Mpa})\end{array}$ \\
\hline DN1000 & - & - & - & 2.96 \\
DN700 & - & - & 1.58 & 1.69 \\
DN500 & 0.90 & 0.95 & 0.92 & 1.01 \\
DN400 & 0.71 & 0.76 & - & - \\
DN300 & 0.57 & 0.60 & - & - \\
DN200 & 0.40 & 0.42 & - & - \\
\hline
\end{tabular}

Remark: The repair length of one point is 4 meter.

Tab. 1-4 The pipe itself repair cost of different pipe diameter（Q235B steel pipe)

\begin{tabular}{cc} 
& {$[$ Ten thousand yuan/ one point } \\
\hline pipe diameter & medium-pressure $(0.4 \mathrm{Mpa})$ \\
\hline DN500 & 1.10 \\
DN400 & 0.90 \\
DN300 & 0.60 \\
DN200 & 0.40 \\
\hline
\end{tabular}

Remark: The repair length of one point is 4 meter.

Tab. 1-5 The pipe itself repair cost of different pipe diameter（20\# teel pipe)

[Ten thousand yuan/ one point]

\begin{tabular}{cc}
\hline pipe diameter & medium-pressure $(0.4 \mathrm{Mpa})$ \\
\hline DN200 & 0.40 \\
DN200 & 0.15 \\
DN80 & 0.13 \\
DN50 & 0.10 \\
\hline
\end{tabular}

Remark: The repair length of one point is 4 meter.

Tab. 1-6 The pipe itself repair cost of different pipe diameter (PE pipe)

[Ten thousand yuan/ one point]

\begin{tabular}{cc}
\hline pipe diameter & medium-pressure $(0.4 \mathrm{Mpa})$ \\
\hline DN300 & 0.50 \\
DN250 & 0.43 \\
DN200 & 0.35 \\
DN110 & 0.30 \\
\hline
\end{tabular}

Remark: The repair length of one point is 4 meter.

Tab. 1-7 The pipe itself repair cost of different pipe diameter (cast iron pipe)

\begin{tabular}{cc}
\hline pipe diameter & medium-pressure $(0.4 \mathrm{Mpa})$ \\
\hline DN700 & 1.93 \\
DN500 & 1.21 \\
DN400 & 0.99 \\
DN300 & 0.66 \\
DN200 & 0.44 \\
\hline
\end{tabular}

Remark: The repair length of one point is 4 meter.

Earthquake damage economic loss calculation of gas pipeline.

In earthquake damage prediction, the economic losses of the gas pipeline are calculated in accordance with the failure points per kilometer. Due to the pipeline excavated the gas pipeline disaster loss calculation formula of one region is as follows:

$$
T=\sum_{i}^{n} \lambda_{i} L_{i} G_{i}
$$


In the formula,

$T$--Total cost of earthquake damage for gas pipeline (yuan),

$\lambda_{i}$--Average damage rate of the i kind of pipe (one point / km);

$L_{i}$--The total length of the i kind of pipe $(\mathrm{km})$;

$G_{i}$--The repair costs of each damage point of the i kind of pipe (yuan)

Earthquake economic loss assessment of gas system as Ningbo an example.

Since 1996, the length of gas pipeline network is totally about 1562 kilometers in Ningbo city. The largest diameter of trunk pipeline is DN300 and generally is around DN200. The sub-high pressure gas pipeline in Tongtu Road started from 3\# pressure regulating station to Airport Road end with length of about 8.5 kilometers, design pressure $0.8 \mathrm{MPa}, \mathrm{DN} 300$ pipe diameter, and steel pipe.

Assuming Tongtu Road is subjected to the seismic of 6 degrees intensity and belongs to ordinary oil. From table 4-1, we know the damage rate of steel pipe in 6 degrees is 0.03 point $/ \mathrm{km}$. The repair cost of each point is $(0.60+0.78) * 1.0=1.38$ ten thousand yuan, so the seismic loss of gas pipeline of $8.5 \mathrm{~km}$ long is $0.03 * 1.38 * 8.5=0.35$ ten thousand yuan. So on, the seismic loss of gas pipeline in Tongtu Road can be given when subjected to the intensity of 7, 8, 9 degree as following table 1-8.

Tab 1-8 The earthquake economic loss prediction of gas pipeline in Tongtu Road [ten thousand yuan]

\begin{tabular}{ccccc}
\hline Intensity & 6 & 7 & 8 & 9 \\
\hline Loss & 0.35 & 4.45 & 12.55 & 58.65 \\
\hline
\end{tabular}

\section{Summary}

By collating earthquake damage data of gas system, the characteristics of earthquake damage are analyzed. The investigations of the pipeline repair cost and the broken road repair cost are carried out in Ningbo City. Then the repair costs for different diameters of different gas pipes are reasonably given. The results provide a reasonable data of earthquake economic losses evaluation for the gas pipeline system.

\section{Acknowledgement}

In this paper, the research was sponsored by Students' science and technology innovation project (Xinmiao talents plan) in Zhejiang province (Project No.2013R422004).

\section{References}

[1] ZengJian Guo,Urban earthquake countermeasures, Beijing, Seismological Press, 1991;

[2] Weiguo Zhou,Seismic damage analysis and disaster reduction countermeasures of City gas pipeline system, The civil construction and environmental engineering, 2009, 31(4), 70-75;

[3] Institute of engineering mechanics, Chinese academy of sciences, Haicheng earthquake damage, Beijing, Seismological Press, 1979;

[4] Jie Li, The lifeline engineering anti-seismic, Beijing, Science press, 2005;

[5]Xiangjian Wang, Endong Guo, Meijing Zhang, Analysis and countermeasures on seismic damages to gas pipeline in Wenchuan earthquake, World Earthquake Engineering, 2012, 28(4).

[6] Ruliang Wang, Underground pipeline damage, aseismic calculation, design and measures, Beijing, Seismological Press, 2007;

[7] Shuzhen Li, Earthquake disaster assessment, Beijing, Seismological Press, 1995. 